\title{
Situación de la tuberculosis multirresistente en Perú
}

\section{Situation of multi-drug-resistant tuberculosis in Peru}

\section{Correspondencia}

H. Oswaldo Jave Castillo rigeljave2008@yahoo.es

Recibido: 15/02/2017

Arbitrado por pares

Aprobado: 31/05/2017

Citar como: Jave HO, Contreras M, Hernández VA. Situación de la tuberculosis multirresistente en Perú. Acta Med Peru. 2017;34(2):114-25

\author{
H. Oswaldo Jave C. ${ }^{1,2, a, b}$, Mariana Contreras M. ${ }^{2, c}$, V. Andrés Hernández U. ${ }^{2, c}$ \\ 1 Hospital Nacional Dos de Mayo. Lima, Perú. \\ 2 Universidad de San Martín de Porres. Lima, Perú. \\ a Médico neumólogo, $b$ docente, $c$ Interno de Medicina
}

\section{RESUMEN}

La historia de las intervenciones y de la evolución epidemiológica de la hiperendemia de la tuberculosis multirresistente (TBMDR) comprende el periodo 1987-2016 y continúa. Un primer problema consistió en admitir su transmisibilidad y la necesidad de medir la magnitud. Durante los años 1990 una subestimación de la magnitud permitió su expansión. Durante los años 2000 se universalizaron las pruebas de sensibilidad, alentadas por la Unidad Técnica de TBMDR del ex-Programa Nacional de tuberculosis del Ministerio de Salud peruano. Durante todo ese lapso se han ensayado diferentes esquemas de tratamiento, con resultados dispares, mostrando persistente eficacia únicamente los esquemas individualizados basados en por lo menos tres drogas nuevas. Gracias al apoyo del Fondo Mundial, los pacientes tuvieron acceso universal al tratamiento. Ambas intervenciones impactaron sobre la tendencia de la hiperendemia, deteniendo su expansión. Limitaciones en los últimos años, incluyendo nuevamente una subestimación del mismo ha permitido su rebrote. Así, Perú es el país con mayor severidad de TBMDR y tuberculosis extensamente resistente en América.

Palabras clave: Tuberculosis resistente a múltiples medicamentos; Tuberculosis; Epidemiología; Perú (fuente: DeCS BIREME).

\section{ABSTRACT}

The history of interventions and the epidemiological history of the hyperendemicity of multi-drugresistant tuberculosis (MDRTB) encompasses the 1987-2016 period, and it still continues. The first problem was to recognize its transmissibility and the need to measure its real magnitude. During the 90 s, the condition expanded because its extent was underestimated. During the first decade of the new millennium, universal access for susceptibility testing was granted, with the support of the Technical Unit of the former National Tuberculosis Control Program of the Peruvian Ministry of Health. During the whole period, different therapy schedules have been tested, with dissimilar results, and only individualized regimens with at least three new drugs were shown to be persistently efficacious. Thanks to the support of the World Fund, patients were able to get universal access to therapy. Both interventions had an impact upon the hyperendemic trend, and the expansion of this was stopped. However, some limitations occurring in the latter years, including an underestimation of the real magnitude of the problem, have led to a rebound in this condition. Consequently, Peru is the country with the most severe cases of MDR TB and extremely resistant tuberculosis in the Americas.

Keywords: Tuberculosis, multidrug-resistant; Tuberculosis; Epidemiology (source: MeSH NLM). 


\section{Introducción}

La tuberculosis multirresistente (o multidrogorresistente, TBMDR, resistente por lo menos a rifampicina e isoniacida) es la forma más severa de tuberculosis (TB), tanto para la salud individual como para la salud pública, por la relativa facilidad de su transmisión a través de la vía aérea. El Perú tiene el mayor número de casos de TBMDR en América, por lo menos desde mediados de los años 1990 hasta la actualidad. Hasta fines de la década de los 90 , organismos y expertos internacionales sostenían que la TBMDR era escasamente transmisible y que podía ser controlada aplicando el DOTS (del inglés: Directly Observed Treatment, Short course) ${ }^{[1-3]}$ y, por tal razón, desalentaban la inversión en tecnología para realizar pruebas de susceptibilidad por los laboratorios nacionales de micobacterias, limitándolas a baciloscopías y cultivo ${ }^{[4,5]}$. En coherencia con ello, no recomendaban a los programas nacionales de los países en desarrollo la implementación de pruebas de susceptibilidad ni tampoco el tratamiento de la TBMDR ${ }^{[5-7]}$. En el año de 1997 el programa mundial de TB de la Organización Mundial de la Salud (OMS) admitió que la TBMDR era igual de transmisible que la cepa sensible y que había que tratarla ${ }^{[8]}$. Paradójicamente, en su guía para manejo de TB fármaco-resistente de 1997 la OMS mencionaba que aun suponiendo una elevada prevalencia de TBMDR no era prioritario su tratamiento sino su prevención ${ }^{[9]}$.

Recién a inicios de los años 2000 la misma OMS admitiría que fue una negligencia no incentivar la adquisición de tecnología para realizar pruebas de susceptibilidad (sensibilidad) a drogas anti TB de primera y segunda línea ${ }^{[10,11]}$.

En consonancia con dichas recomendaciones, los programas de TB de los países en desarrollo se desatendieron de enfrentar la epidemia creciente de TBMDR ${ }^{[9-14]}$. Tal fue el caso de Perú, que en pleno crecimiento de la epidemia de TBMDR a mediados de los años 1990, solo admitía la existencia de apenas 270 casos $^{[15,16]}$ cuando el laboratorio de micobacterias del Instituto Nacional de Salud peruano (INS) mostraba que estaba detectando más de 1 000 pacientes ${ }^{[17]}$.

En el Perú aún no es posible realizar una revisión sistemática de la bibliografía sobre la epidemiología de la TBMDR publicada en el país porque no toda la información ha sido publicada en revistas vigentes, muchas se han publicado en revistas institucionales hospitalarias ya fenecidas, otras no están indexadas ni registradas en bases de datos electrónicas. Además, la principal fuente de información (la Unidad Técnica de TBMDR [UTTBMDR] del exPrograma de TB del Ministerio de Salud [MINSA]) publica de manera intermitente solo presentaciones en diapositivas (solo entre 2006 y 2010 la UTTBMDR publicaba boletines estadísticos electrónicos de TB y TBMDR). Por otra parte, el Programa Nacional de Control de TB (PNCT) publicaba anuarios de TB hasta el año 2005. Asimismo, se revisaron los artículos sobre los estudios nacionales de prevalencia de resistencia a drogas anti-TB de 1996, 1999 y 2006. Es de mencionar que la base de datos electrónica de TBDR (TB drogoresistente) y TBMDR de la UTTBMDR de Perú es probablemente la más poderosa del mundo en cuanto a información y cantidad de datos, a punto que mereció un premio nacional de tecnología en EE. UU. por ser la mejor base electrónica de TBMDR del mundo y haber sido desarrollada por un grupo de médicos de la Universidad de Harvard (Partners in Health) y del Instituto Tecnológico de Massachusetts ${ }^{[16,18-21]}$.

De cómo se inició la epidemia de TBMDR en el Perú, su evolución y los resultados del tratamiento anterior, actual y futuro trata esta revisión. No incluye tópicos importantes como TBMDR y comorbilidades (VIH, diabetes, cáncer), TBMDR en trabajadores de salud, control nosocomial de TBMDR, derechos humanos y sociedad civil ni tuberculosis extensamente resistente (TBXDR).

\section{El contexto precedente al inicio de la epidemia de TBMDR}

Las primeras pruebas de sensibilidad (susceptibilidad) para detectar TBMDR se iniciaron a mediados de los años 1980 en el hospital Sergio Bernales del distrito de Collique-Lima y en el INS. En el año 1985 se detectaron 60 casos y el año 1990, 59 casos $^{\text {[22] }}$. Luego se publicaría el estudio de resistencia primaria del periodo 1993-1994, con mejor diseño y representatividad, incluyendo 477 pacientes nuevos al tratamiento, de Lima y regiones. En este estudio encontraron una resistencia inicial de $0,8 \%$ a rifampicina $(\mathrm{R}), 5,5 \%$ a isoniacida $(\mathrm{H})$ y en ningún caso resistencia a ambas de manera simultánea ${ }^{[23]}$. En sentido estricto se trataba de casos de TB polirresistente (resistente por lo menos a dos drogas, no necesariamente casos de resistencia simultánea a R-H, como es la definición actual). Es necesario precisar que la definición de TBMDR recién se estandarizó en el año 1994, hasta ese entonces, la TBMDR y TB polirresistente eran sinónimos ${ }^{[24]}$. El Seguro Social del Perú (en ese entonces Instituto Peruano de Seguridad Social) inició el uso de esquemas modernos en 1978, incluyendo R, I y pirazinamida (Z). El MINSA empezó a utilizar rifampicina en el año 1985 como parte de un estudio piloto y recién utilizó dicha droga a escala nacional desde el año $1991^{[25]}$.

En el periodo 1980-1991 el porcentaje de tratamiento irregular y abandono del mismo era elevado ( $41 \%$ y $34 \%$ en las cohortes de 1980 y 1981, respectivamente, con un tratamiento autoadministrado, principalmente), de manera que es posible que en dicho periodo aparecieran los primeros casos de TBMDR, en forma de recaídas, abandonos recuperados y fracasos al tratamiento ${ }^{[26]}$. A los dos primeros grupos se los trato desde 1991 con el entonces esquema único (2RHZE/4(R2H2)), luego llamado esquema UNO desde el año 1996, incrementando con ello el riesgo de ampliación de la resistencia y la transmisión de la misma. A los últimos (los fracasos) se les brindaba un retratamiento débil, que incluía a la kanamicina $(\mathrm{Km})$ y Diateben (D, es la combinación de Tioacetazona e H), más las tres drogas a las cuales ya había fracasado: $R+Z+$ etambutol $(E)$, a pesar de que el programa de TB de Perú ya disponía de evidencia de que se trataba de pacientes con TBMDR ${ }^{[27]}$. Con dicho esquema el porcentaje de curación era menor de $60 \%{ }^{[28-30]}$, aunque los consultores oficiales del programa nacional de TB reportaron hasta $80-85 \%$ de curación ${ }^{[31,32]}$. De ser cierto, habría detenido la epidemia de TBMDR, pero luego de un cuestionamiento a la veracidad de dicha información por parte del oficial visitante de OMS en 1995, el esquema fue abandonado ${ }^{[6,33]}$. 
En el año 1993, un grupo de médicos y enfermeras de ComasCollique presentó la primera investigación operativa de cohortes de tratamiento estratificadas (nuevos, recaídas y abandonos recuperados) ${ }^{[34]}$. Hasta entonces el programa nacional solo presentaba una cohorte de tratamiento nacional: la de casos nuevos más los casos con recaídas, juntos. El estudio mostró que dicho esquema producía muchos fracasos en el caso de los pacientes que ingresaban con la categoría "recaídas" (una diferencia estadísticamente significativa, lo que sugiere que las recaídas de entonces encubrían los casos de TBMDR). En aquellos años el programa nacional estigmatizaba a los programas de TB locales que reportaban una alta tasa de fracasos al esquema UNO, por lo que algunos establecimientos de salud preferían darlos de alta como curados e inmediatamente los reingresaban como "recaídas" suponiendo, con ello, que sí se curarían en esta segunda vez. Esta conducta se explicaba porque, según la versión oficial del programa nacional, los fracasos al tratamiento eran consecuencia del tratamiento autoadministrado ${ }^{[35]}$. Por tanto, suponían que volviéndoles a dar el mismo esquema, pero supervisándolos, se curarían. Pero aquella era también la opinión de la UNION (Unión Internacional Contra la TB y Enfermedades
Respiratorias) ${ }^{[36]}$. Esa conducta lo que producía más bien era casos con resistencia ampliada, preludio a la aparición de casos de TBXDR.

En el año 1996 el programa nacional decidió que los casos de fracaso al esquema único debían recibir lo que se denominó esquema dos y posteriormente esquema dos reforzado ${ }^{[37]}$, que consistía en repetir las cuatro drogas a las que ya había fracasado el paciente (RHZE), agregándole una única droga nueva (estreptomicina, S): 2RHZES/1RHZE/5(RHE)2, una verdadera monoterapia encubierta ${ }^{[38]}$, cuando la recomendación internacional era tratar estos pacientes con al menos tres drogas nuevas ${ }^{[39]}$. El primer consolidado de la cohorte de tratamiento con esquema DOS para los pacientes que habían previamente fracasado al esquema uno mostró un $30 \%$ de fracaso reiterativo, pero el programa anuló dicha información y ordenó volver a consolidar una nueva cohorte de tratamiento que dio lugar a un reporte de fracaso de $4,5 \%{ }^{[33]}$, porcentaje que fue cuestionado por expertos nacionales de la Sociedad Peruana de Neumología (SPN, en reunión formal con el PNCT y la Organización Panamericana de la Salud; Dr. Jave, comunicación personal) y por

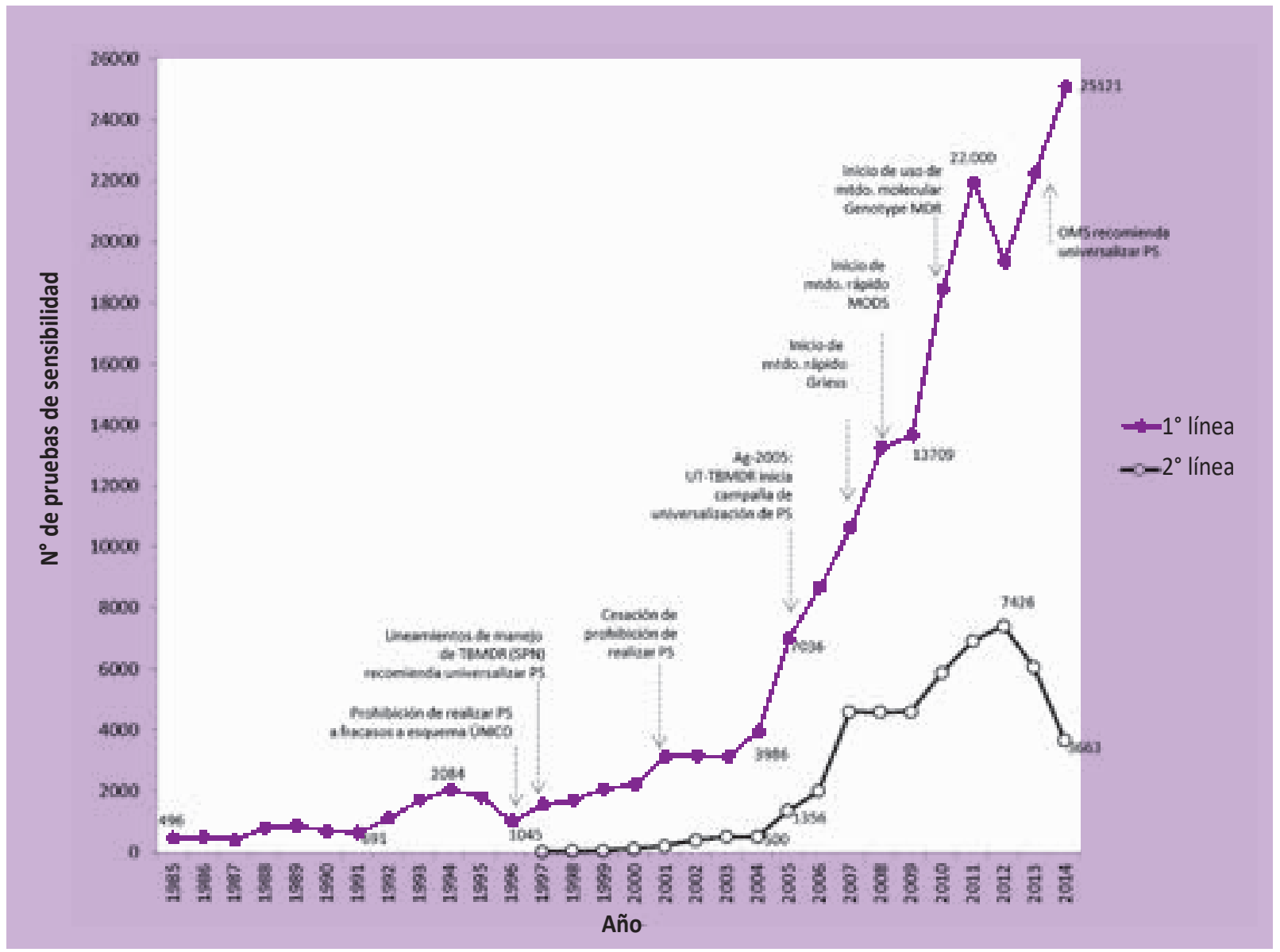

Fuente: PNCT, ESNPCT, UT-TBMDR, Luis Asencios, et al, elaboración propia.

Figura 1. Número de pruebas de sensibilidad (PS) a drogas anti-TB de primera y segunda línea. Se muestran las fechas aproximadas de incorporación de pruebas rápidas y las moleculares y se observa una tendencia descendente en la realización de pruebas de segunda línea en los últimos años, los que pueden influenciar sobre el reporte oficial de casos de TBXDR. 


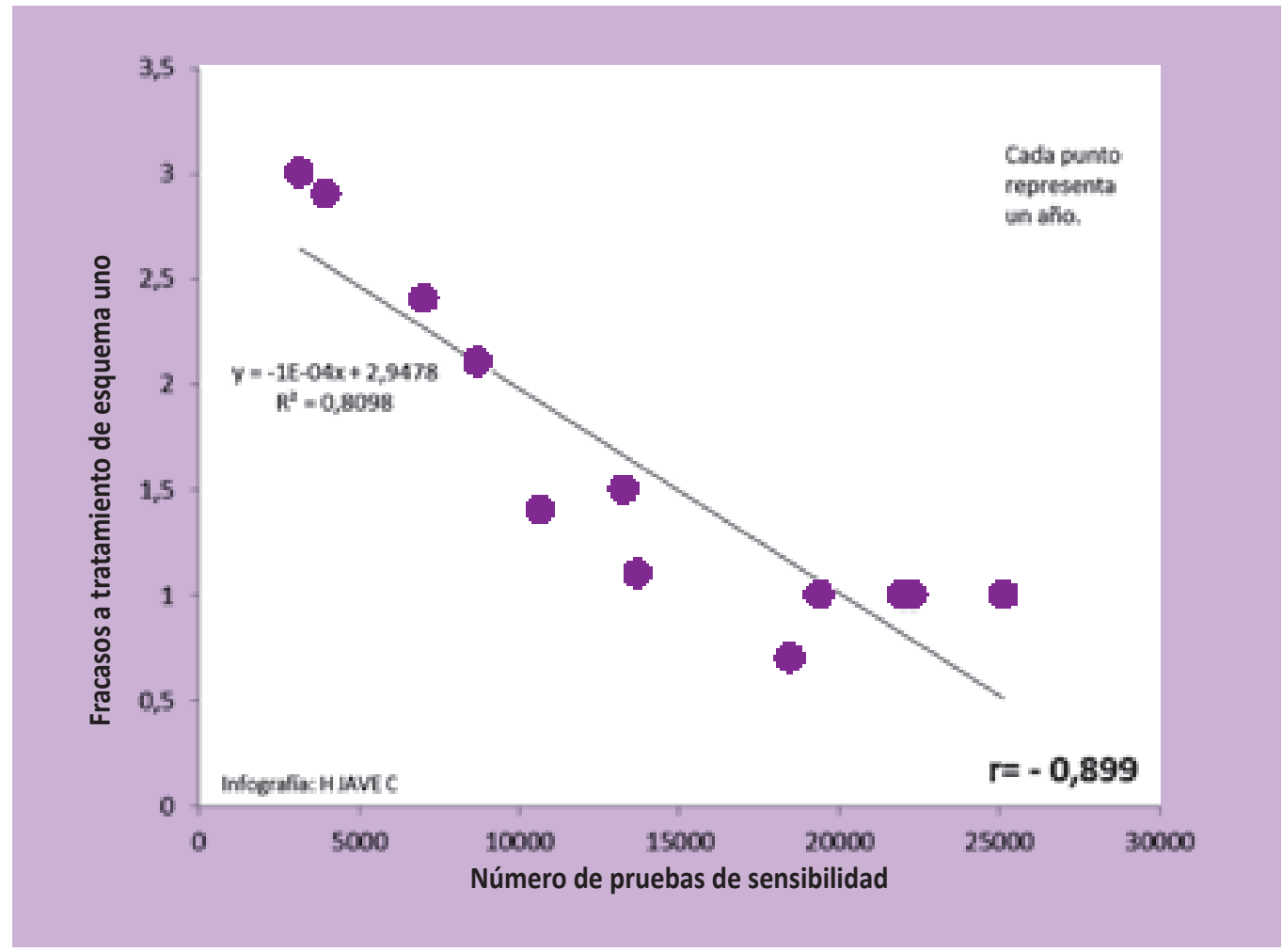

Fuente: basado en información de la ESNPCT.

Figura 2. Correlación entre número de pruebas de sensibilidad (de $1^{\circ}$ línea) y fracasos a tratamiento con (2RHZE/4R2H2). Se muestra una elevada correlación negativa $(r=-0,89)$ entre los fracasos al tratamiento de esquema UNO y el incremento de las pruebas de sensibilidad (de $1^{\circ}$ línea). Cada punto representa una cohorte de tratamiento (en total 12 cohortes anuales de tratamiento con esquema UNO: 2RHZE/4R2H2).

el informe de la OMS al MINSA, sugiriendo una subestimación de la verdadera magnitud de los fracasos ${ }^{[6,40]}$. Luego los fracasos seguirían incrementándose, hasta llegar a 70\% (información reconstruida con los informes operacionales regionales) ${ }^{[40]}$. Para el segundo lustro de la década de los años 1990 el laboratorio de micobacterias del INS reportó un incremento sostenido de los casos de resistencia simultánea a cinco drogas, que sería el preámbulo a la aparición de los primeros casos de TBXDR ${ }^{[17]}$.

En los años 1990, 1996 y 2006 se realizaron tres estudios nacionales de prevalencia de TB resistente entre casos nuevos y antes tratados de TB. Los estudios nacionales de resistencia calculan un tamaño de muestra apropiado basado en la prevalencia estimada de resistencia a isoniacida ${ }^{[41-43]}$. Para el caso de los antes tratados, simplemente se acumulan los casos según se vaya completando la cuota de casos nuevos programados para el estudio. Así, el más importante indicador es la prevalencia de TBMDR en los casos nuevos de TB pulmonar frotis positivo (TBPFP) ${ }^{[27]}$. Los hallazgos muestran cómo se fue incrementando la prevalencia de TBMDR inicial en el curso de dichos años. Los porcentajes del año 2006 tenían dos valores diferentes: el publicado oficialmente por el INS (5,8\% de prevalencia de TBMDR primaria) basado en los resultados de las pruebas de laboratorio y las fichas de recolección de información y el segundo porcentaje $(3,8 \%)$ correspondiente al estudio de campo realizado por el
ex-Programa de TB basado en la revisión de las historias clínicas (para detectar si se tratan de verdaderos casos nuevos o si son casos antes tratados erróneamente calificados como nuevos) y su contraste con las pruebas de sensibilidad respectiva. Esta última metodología es la que permite finalmente establecer la verdadera magnitud de la resistencia inicial. En cualquier caso, la TBMDR primaria estaba en incremento.

\section{El esfuerzo por detectar TBMDR}

En los países desarrollados, a pesar de haber iniciado el uso de esquemas modernos basados en la rifampicina a mediados de los años 1970; sin embargo, las tasas de prevalencia de TBMDR en casos nuevos siempre habían sido bajas, a pesar de haber brindado tratamiento autoadministrado en aquellos años (el tratamiento supervisado en gran escala recién se extiende a fines de los años 1990). La razón subyacente probablemente sea que la práctica médica común en estos países solicita prueba de sensibilidad desde el inicio de tratamiento ${ }^{[44]}$, contrario a lo que ocurre en los países en desarrollo, que tienen acceso restringido a pruebas de sensibilidad, y que las realizan luego de haber fracasado a un primer tratamiento (o como el caso del Perú, que entre 1996 y 2000, prohibió realizarlas y permitirlas solo cuando fracasaran dos veces consecutivamente siempre que los pacientes siguieran vivos) ${ }^{[45,46]}$. 
Desde mediados de la década de 1980, el Perú tenía una capacidad limitada para realizar pruebas de sensibilidad. Desde el segundo lustro de los años 1990 se fue incrementando la demanda de pruebas de sensibilidad, a pesar de la prohibición de realizarlas. La ONG Socios en Salud (en inglés: Partners in Health) apoyaba esta actividad de manera discreta, enviando las pruebas al Laboratorio de Tuberculosis de Boston, Massachussett.

La prohibición se levantó el año 2001 pero aún en aquellos años era necesaria la firma del neumólogo o del consultor del CERI (Comité de Evaluación de Retratamiento Intermedio) para aceptar realizar una prueba de sensibilidad e ingresar a tratamiento TBMDR ${ }^{[47,48]}$. Sin embargo, en agosto del año 2005, la UTTBMDR del programa de TB de MINSA convocó a los neumólogos consultores nacionales, para proponerles universalizar las pruebas de susceptibilidad (PS) y dejar por fin de tratar a ciegas a los pacientes con TB, obteniéndose la aprobación por unanimidad (O. Jave, exresponsable de la UTTBMDR, comunicación personal). Así, el proceso de universalización, en el que todo médico o enfermera del programa de control de TB de atención primaria podía solicitar la prueba desde el momento del diagnóstico, se aceleró: en un solo año (2005) las PS se incrementaron en $44 \%$ y para el año 2011 se había multiplicado en más de $500 \%$, siendo el país con mayor número de PS en América Latina (Figura 1).

En el año 2007 se empezó el uso de pruebas rápidas en América Latina en condiciones de programa, iniciado en el Perú con el método de nitrato-reductasa (método GRIESS) [47], seguido años después del MODS ${ }^{[48]}$ ambos entregando resultados en 30 días. $A$ partir del año 2010 iniciamos en el Perú el uso de pruebas rápidas moleculares en condiciones de programa, siendo también la primera vez en América Latina (Genotype PlusMDRTB) ${ }^{[49]}$, cuyos resultados podrían entregarse en menos de ocho horas, pero que sin embargo, por problemas operacionales del INS, se entregan actualmente después de dos semanas, por lo que se ha iniciado un proceso de descentralización del método.

Desde el año 2005, el número de PS a drogas de segunda línea se incrementó en casi 300\% (de 500 el año 2004 a 1356 el año 2005), preparándonos para la detección de TBXDR ${ }^{[50,51]}$.

\section{La situación de la TBMDR}

Conforme se fueron universalizando las pruebas de sensibilidad desde el inicio de tratamiento, los fracasos al esquema UNO (2RHZE/4R2H2) fueron disminuyendo rápidamente,

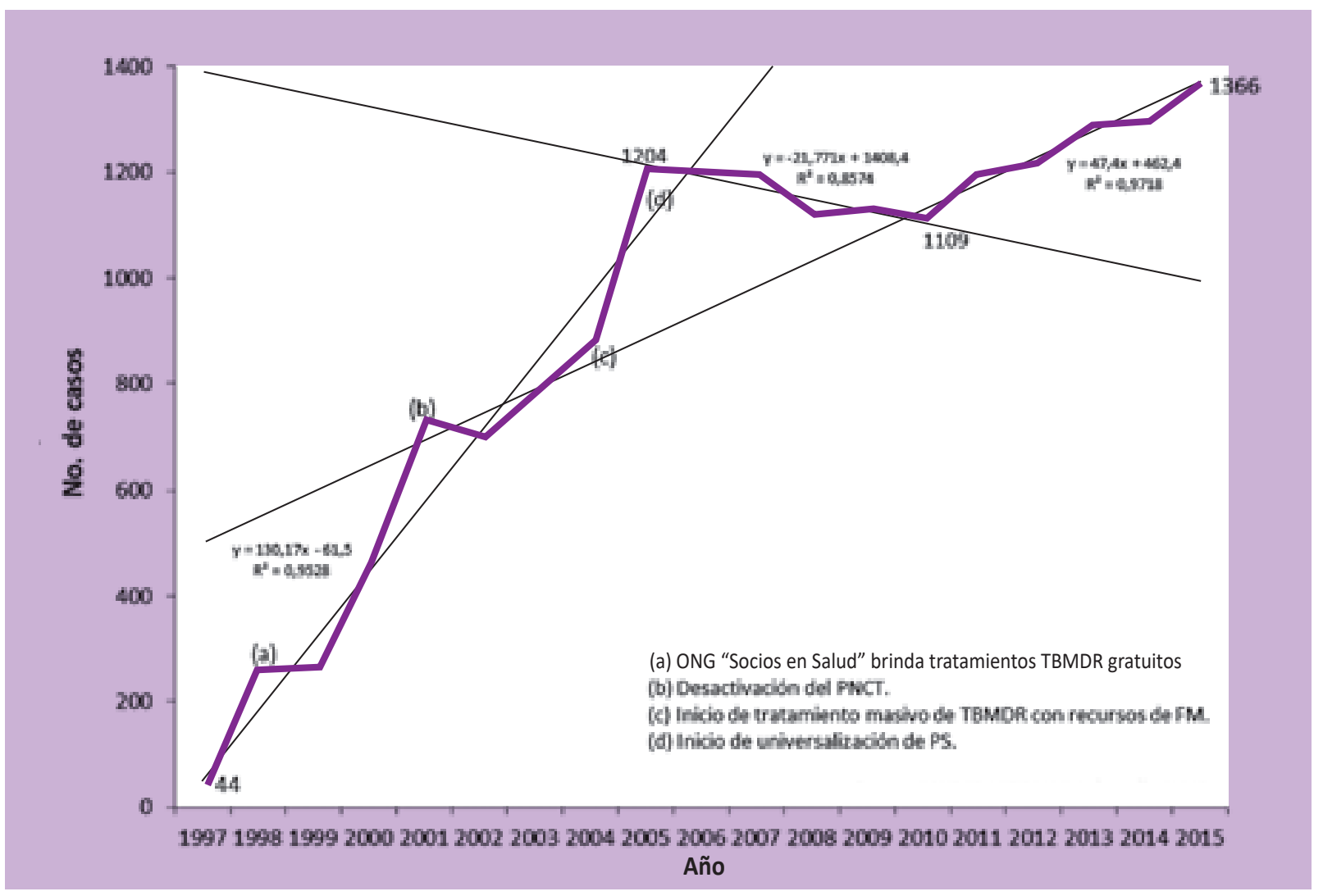

Fuente: PNCT, ESNPCT, UT-TBMDR, Luis Asencios et al, elaboración propia.

Figura 3. Evolución del número de casos de tuberculosis multirresistente (TBMDR) y líneas de tendencia. Se muestra la evolución anual del número de casos de TBMDR en Perú, desde el año 1997 hasta el año 2015. Se observa una primera fase ascendente, una segunda fase de detención del incremento seguido de una meseta epidemiológica y una tercera fase de renovado incremento de casos. PNCT: Plan Nacional de Control de Tuberculosis; FM: PS: prueba de sensibilidad; FM: fondo mundial.. 
observándose una fuerte correlación negativa entre el incremento de PS de primera línea y fracasos al tratamiento con dicho esquema $(r=0,899){ }^{[52]}$ (Figura 2) Ilegando a disminuir este a $0,7 \%$, pero es muy probable que el porcentaje verdadero de fracasos sea mucho menor pues, tal como ocurre operacionalmente, esta proporción de fracaso reportado incluye casos de micobacterias atípicas erróneamente diagnosticados y tratados como TB, casos erróneamente calificados como fracasos cuando en realidad son conversiones tardías pansensibles, casos reportados como fracasos al tratamiento al segundo y tercer mes, los que no corresponden a la definición aceptada de cuarto mes con cultivo negativo, así como nueva reinfección en el curso de un tratamiento exitoso. Por último, incluye también casos de tratamiento autoadministrado irregularmente, lo que nos sugiere que el verdadero aporte de los casos irregulares a la carga de TBMDR en Perú debe ser proporcionalmente muy bajo. La cohorte nacional de tratamiento de TB pulmonar pansensible del año 1999 mostró una proporción de fracasos de 0,6\% ${ }^{[40]}$.

La evolución de la TBMDR en Perú ha tenido cuatro etapas (Figura 3):

La primera etapa (1991-1996) en la que la información sobre el verdadero número de casos de TBMDR es muy escasa y no está registrada en el sistema de información operacional del PNCT, contando únicamente con la información con limitaciones del INS.

Una segunda etapa, (1997-2005), en el que se triplican los casos de fracasos al tratamiento de esquema UNO (de 1,0\% a $3,0 \%$ ), se incrementa rápidamente las pruebas de sensibilidad (de 1602 hasta 7 036) y se llega al máximo en el número de casos confirmados de TBMDR (de 44 a 1204 ). Esta etapa fue de incremento exponencial epidémico de casos reportados, siendo probablemente una mezcla de varios factores: la suma de errores del PNCT de subestimar la magnitud de la TBMDR, poner en circulación esquemas de tratamientos para TB recomendados por OMS y la UNION (esquemas DOS y luego DOS REFORZADO) y para TBMDR (estandarizado antiguo y estandarizado nuevo para TBMDR), que incrementaron los fracasos y como consecuencia el tiempo de transmisión de TBMDR, lo que llevó a un incremento real de casos, lo que sugiere que estaba ocurriendo una transmisión acelerada de TBMDR, principalmente en el escenario domiciliario (con fuerte hacinamiento y escasa ventilación, como sugieren los estudios nacionales respecto a la calidad de la vivienda) pero también en otros escenarios de concentración pública. Otro factor contribuyente muy importante fue la desactivación del PNCT, transfiriéndose las actividades de control de TB a una amorfa oficina denominada Componente TB, desmembrada de sus funciones, con reducción severa de su personal (de 12 funcionarios en el nivel central a dos médicos generales) y reducción del $50 \%$ del presupuesto destinado a sus actividades. Ello llevó a un desabastecimiento de insumos de laboratorio; por tanto, menor número de cultivos para Mycobacterium tuberculosis y consecuentemente una detención en la producción de PS; asimismo llevó a la práctica desactivación de su sistema de información y casi nulas actividades de capacitación y supervisión a los niveles regionales y locales. Es en este escenario que se reportaron los primeros casos de resistencia a nueve drogas, casi con seguridad se trataba de casos de TB extensamente resistente (TBXDR). Ningún otro país en el mundo, con similar severidad de endemia de TB y con una epidemia en curso de TBMDR, había cometido tal error de salud pública. Por último, es recién a partir del año 2005 que el Estado Peruano empieza a comprar medicamentos para TBMDR con apoyo del Fondo Mundial (hasta entonces solo se contaba con el apoyo de una ONG para menos de 200 pacientes, insuficiente para detener la epidemia).

Una tercera etapa de meseta o aplanamiento de la epidemia (2006-2010), en el que se detiene el incremento en el número de casos de TBMDR (de 1204 a 1145 casos), a pesar de incrementarse la búsqueda de casos a través de las PS. Ocurre además una caída máxima de los fracasos al tratamiento con esquema UNO (de 3,0\% a 0,7\%, aunque en la cohorte de casos de TBPFP pansensible la proporción de fracasos apenas llegó a $0,6 \%$, un porcentaje aceptado internacionalmente y muy cercano a los resultados de estudios en condiciones controladas de ensayo clínico). En este periodo se incorporaron nuevas pruebas de sensibilidad rápidas a las actividades del PNCT (GRIESS y MODS) y pruebas moleculares (Genotype MDRTBplus). Hasta el año 2005 había que esperar que el paciente fracasase a tratamiento con drogas de primera línea para recién solicitar PS ${ }^{[53]}$. En agosto del 2005 la UTTBMDR lanzó la campaña de universalización de las PS. Asimismo, se universaliza el acceso al tratamiento gratuito de TBMDR, siendo desde entonces el Estado peruano el que se encargaba de la totalidad de compra de medicamentos.

Una cuarta etapa, (2011 hasta la actualidad), ocurriendo un nuevo rebrote de casos de TBMDR ${ }^{[54,55]}$. Coincide con un cambio en la gestión de la Estrategia Sanitaría Nacional para el Control de Tuberculosis (ESNPCT), cambio en el esquema de tratamiento de casos nuevos de TB e incremento continuo de las PSy del número de laboratorios que realizan pruebas rápidas, aparentemente sin control de calidad para algunos de estos. En julio del 2011 la ESNPCT logró el apoyo de la casi unanimidad de médicos del comité de petitorio de medicamentos de la DIGEMID para aprobar la compra de Linezolid (Lzd) para iniciar el tratamiento de TBXDR, con fuerte oposición de los químico-farmacéuticos, adquiriéndose Lzd para 50 pacientes aproximadamente. En el periodo 2010-2014, se observó un incremento exponencial en la monoresistencia a isoniacida y un incremento a menor velocidad de la monoresistencia a rifampicina ${ }^{[55]}$ (Figura 4). En ambos casos ello predispone a fracaso de tratamiento de esquema primario, de ser el caso, y la ampliación de la resistencia a TBMDR. Eso explicaría, parcialmente, el fenómeno que se está observando desde el año 2012: un nuevo incremento sostenido de los casos de TBMDR, cuando lo que se esperaba después de la meseta epidemiológica era una disminución rápida y sostenida de la TBMDR, como ha ocurrido en otros países con severa epidemiaendemia de TBMDR ${ }^{[56,57]}$. Coincide también con un periodo de incremento espectacular de los abandonos de tratamiento TBMDR y con un incremento de la prevalencia de TBMDR inicial según el cuarto estudio nacional de resistencia a medicamentos 


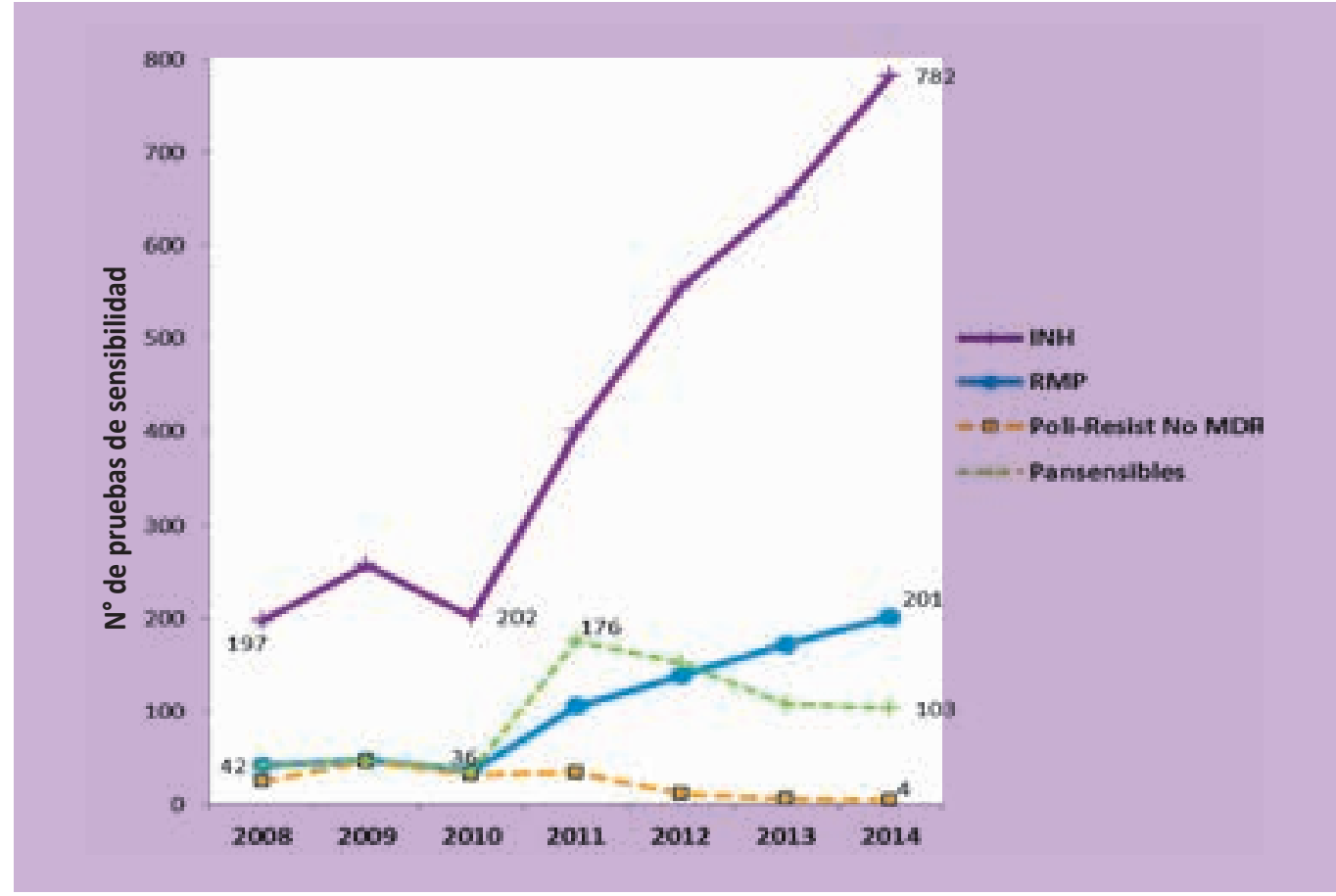

Fuente: ESNPCT, UT-TBMDR.

Figura 4. Evolución de la resistencia no MDR a drogas antituberculosas. Se muestra la tendencia anual de la tuberculosis monorresistente a diferentes drogas de primera línea entre los años 2008 y 2014. Se observa que la resistencia a isoniacida (INH) se está incrementando de forma exponencial, separándose de la resistencia a rifampicina (RMP). Poli-Resist No MDR: polirresistencia no multidrogorresistente.

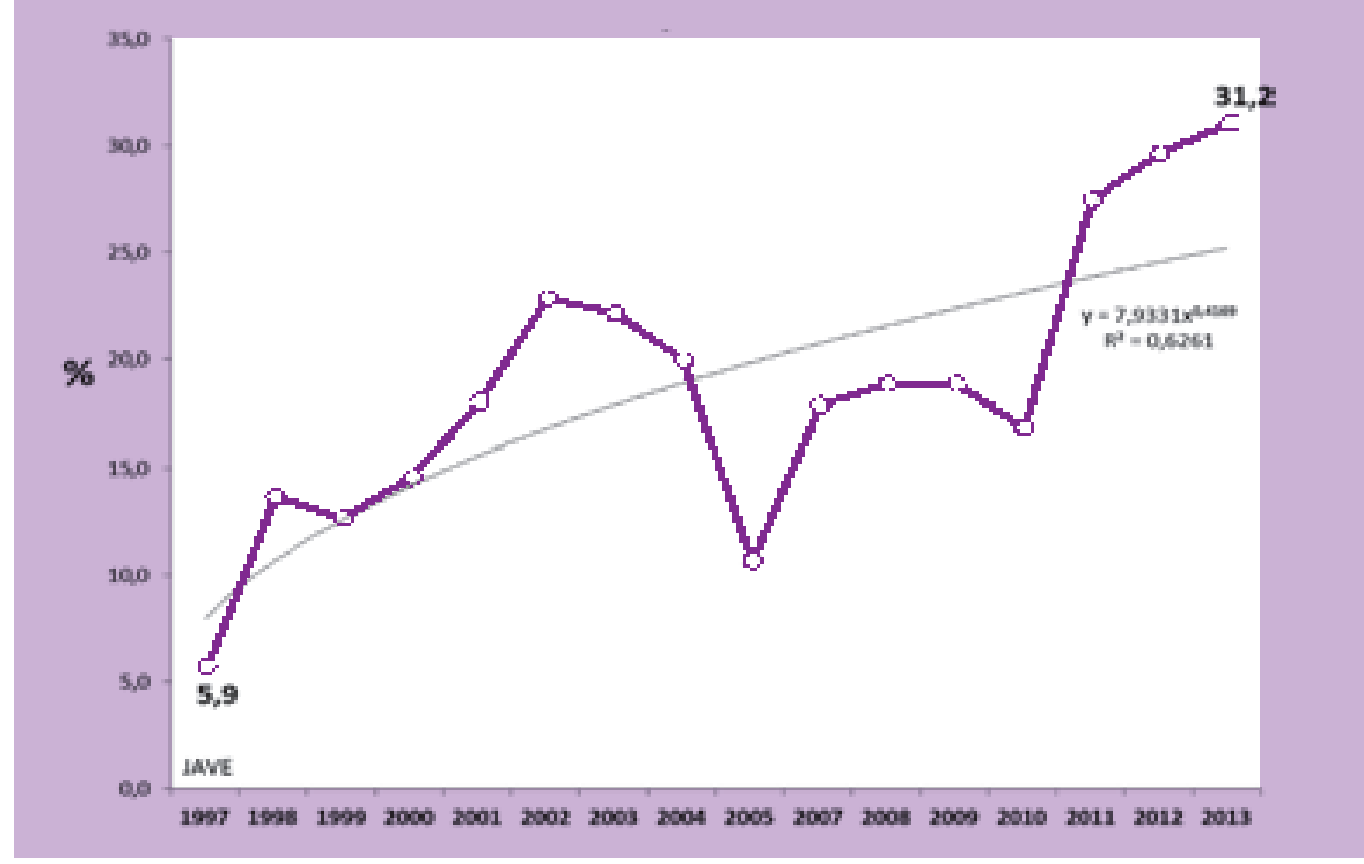

Nota: no se dispone de información respecto al año 2006

Fuente: PNCT, ESNPCT, UT-TBMDR, elaboración propia.

Figura 5. Abandono de tratamiento TBMDR (\%) según cohorte de ingreso a tratamiento. Se muestra como se ha ido incrementando el abandono, de 5,9\% en la cohorte de ingresantes el año 1997, hasta 31\% en la cohorte ingresante al año 2013 (un incremento de 500\%). Dado que el tratamiento dura entre 18 y 24 meses, solo se puede consolidar la información al final de haber cumplido los dos años de tratamiento. Por tanto los años identifican el año en que ingresaron a tratamiento para la tuberculosis multirresistente (TBMDR). 
anti-TB del año 2014-2015 (aún no publicado), lo que sugiere un debilitamiento grave de las actividades operacionales del nivel central de la ESNPCT en los últimos años (Figura 5).

\section{La calidad de la información sobre TBMDR}

En el periodo 1997 hasta el año 2001 se exigía estrictamente una PS que demostraba la condición de TBMDR para acceder al tratamiento con el esquema de entonces. Aun así, la mayoría de pacientes no tenía acceso a tratamiento TMDR gratuito (cobertura de apenas 28\% para el periodo 1994-1999 con esquemas muy débiles) ${ }^{[58]}$.

Desde el año 2001 hasta el año 2005 los pacientes ingresaban a tratamiento con una simple solicitud no estandarizada, vía internet, del neumólogo en el que indicaba que se trataba de un paciente con sospecha de TBMDR. A partir del 2005 se modificó esta forma de requerimiento y se reactivaron, capacitaron, evaluaron y expandieron los comités regionales de expertos en TB/TBMDR (CERIS), los que decidían quienes debían acceder al tratamiento, siempre basado en la PS y ya no en simple sospecha, con algunas excepciones (niños, gestantes, diabéticos y casos de VIH/TB). La UTTBMDR hizo una recomposición de la información disponible en la base de datos electrónica (RME, registro médico electrónico, probablemente la base de datos más potente del mundo en ese entonces y que sirvió de modelo para otros países como Brasil y México, Filipinas, Rusia, etc.). Dicha actividad permitió conocer quiénes eran los que ingresaban a tratamiento TBMDR. Así, se llegó a determinar que cada año se iba incrementando el ingreso a tratamiento TBMDR a pacientes que realmente no lo eran y no tenían siquiera una PS. El año 2005 , por ejemplo, solo el $66,6 \%$ de los ingresados a tratamiento tenían PS (contra más del $90 \%$ en el caso de EEUU). Además, solo el $49,4 \%$ de los ingresados a dicho esquema eran realmente casos de resistencia por lo menos a R-H (Figura 4).

\section{El tratamiento de la TBMDR (Tabla 1)}

Las primeras cohortes de tratamiento de TBMDR, hasta donde estamos informados, fueron presentados por el Dr. E. Félix, jefe del Servicio de Neumología del Hospital E. Rebagliati de la Seguridad Social en el Congreso Mundial de la Unión Internacional Contra la TB, el año 1992, reportando más de 200 pacientes, con esquemas individualizados, con una tasa de curación de $80 \%$ aproximadamente ${ }^{[59]}$. Por parte de MINSA se presentaron dos cohortes de tratamiento TBMDR estandarizados, en el Congreso Mundial de la UNIÓN Internacional Contra la TB, en Lima en 1994, con esquema 4KmDRZE/8DRZE, obteniendo según los autores más de $80 \%$ de curación ${ }^{[31]}$. Sin embargo, una cohorte de tratamiento de TBMDR con dicho esquema, correspondiente a 350 pacientes atendidos en el hospital de Collique mostró resultados totalmente discrepantes de los resultados antes mencionados: una curación de menos de $50 \%$ y una recaída a dos años de $25 \%{ }^{[29]}$. Las misiones posteriores de OMS recomendaron dejar de utilizar este esquema oficial antiguo del PNCT, por su baja eficacia, lo que sugiere una sobreestimación de las tasas de curación ${ }^{[6]}$. Este esquema dejó de utilizarse el año 1997, empleándose un nuevo esquema estandarizado: $3 \mathrm{KmCxEtoHZE/15KmCxEtoZE} \mathrm{(Cx:} \mathrm{ciprofloxacino;}$ Eto: etionamida), también con magros resultados (curados: 4454\%, fracasos: $23-37 \%)^{[60,61]}$ (Tabla 1).

Paralelamente, la ONG Socios en Salud fue autorizada a brindar tratamiento individualizado, inicialmente a menos de 70 pacientes y luego a más de 200 , obteniendo excelentes resultados, con escasa tasa de abandono de tratamiento (menor de $3 \%$ ) y menor tasa de fallecidos. Así, el porcentaje de fallecidos fue disminuyendo sostenidamente, hasta llegar a 6,0\% ${ }^{[62]}$.

Es importante mencionar lo que sucede cuando se utilizan esquemas de tratamiento con baja eficacia ( $50 \%$ o menos): las tasas anuales de prevalencia se modifican muy poco y disminuyen los fallecidos, por lo que se incrementa el número de transmisores crónicos de TBMDR, creándose un equilibrio perverso que endemiza el problema ${ }^{[63]}$.

En 1997 la SPN editó el texto Lineamientos de Manejo de la TB Multirresistente ${ }^{[64]}$ en la que recomendaba universalizar las PS y tratar a los pacientes con por lo menos tres drogas nuevas nunca antes utilizadas en el paciente. En el curso de los años 1990, como consecuencia de la escasa experticia en el manejo de la TBMDR por el equipo de médicos de atención primaria que manejaba el nivel central del PNCT, se subestimó la magnitud de la epidemia y se maltrató con esquemas inapropiados que en lugar de detener la epidemia de la TBMDR la amplificaron y extendieron en complejidad, dando lugar en el segundo lustro a la más grande hiperendemia de TBMDR que hubiera conocido el país y creando el substrato para la aparición de la TBXDR en el segundo lustro. Inicialmente trató la TBMDR con esquema primario (esquema UNO y luego esquema DOS, luego Esquema DOS REFORZADO). Luego a partir de 1997 se aplicó un esquema ESTANDARIZADO para TBMDR que incluía, además de la kanamicina (que se estuvo aplicando desde 1991) al ciprofloxacino y ethionamida. Este esquema estandarizado, recomendado por OMS, volvió a mostrar ineficacia, obteniendo bajas tasas de curación, con rangos entre $46 \%$ y $79 \%$ (promedio $66 \%$ ), con porcentajes de abandono de tratamiento crecientes. A partir del 2005, gracias a recursos aportados por el Fondo Mundial, el MINSA pudo universalizar el acceso a tratamiento para TBMDR. Hasta entonces la cobertura de tratamiento apenas llegaba a 28\%, con lo que era imposible detener la epidemia en curso, más aún si se había desactivado el programa de TB y desfinanciado sus actividades. A partir del 2005 la ESNPCT decidió aplicar tres esquemas diferenciados: estandarizado (sin necesidad de prueba de sensibilidad), (4-6KCxEtoCsZE/14-18CxZEEtoCsPAS), empírico (basado en la prueba de sensibilidad del caso que contagia) e individualizado (basado en la prueba de sensibilidad del propio paciente). Las curvas de conversión bacteriológica, basadas en cultivos mensuales, mostraron una diferencia estadísticamente significativa a favor del esquema individualizado, que es la forma como se trata la TBMDR en los países desarrollados ${ }^{[65]}$.

Una calificación estricta del caso TBMDR, la universalización de las pruebas de susceptibilidad y el acceso temprano a tratamiento 
Tabla 1. Cohortes de tratamiento de TBMDR, según año de ingreso a la cohorte.

\begin{tabular}{|c|c|c|c|c|c|c|c|c|}
\hline Esquema & Cohorte & $\begin{array}{c}\mathbf{N}^{\circ} \\
\text { pacientes }\end{array}$ & Curados & Fracasos & Fallecidos & Abandonos & $\begin{array}{l}\text { Transfer. } \\
\text { S/C }\end{array}$ & Recaídas \\
\hline 2RHZE/4(RH)2 & Perú 1996 & 24 & 54,1 & 25,0 & 17,0 & 0,0 & 0,0 & 0,0 \\
\hline 1RHZES/1RHZE/5(RHE)2 & Nacional 1996 & 118 & 85,0 & 6,0 & 12,0 & 11,0 & 4,2 & $S / I$ \\
\hline 1RHZES/2RHZE/5(RHE)3 & Comas 1997-1998-2000 & 69 & $8,7-18,1$ & $60,6-86,9$ & $4,3-15,0$ & $S / I$ & $S / I$ & $S / I$ \\
\hline 4KDRZE/ 8DRZE & Callao & 241 & $51,6-81,1$ & 18,9 & $S / I$ & $S / I$ & $S / I$ & $S / I$ \\
\hline 4KCxDRZE/ 8CxDRZE & Perú 1991-1996 & $S / I$ & 53,4 & 24,3 & 10,8 & 11,5 & 0 & $S / I$ \\
\hline 4KDRZE/ 8DRZE & $\begin{array}{c}\text { Comas-Indep-Collique } \\
\text { 1991-1995 }\end{array}$ & 105 & 54,2 & 26,6 & 4,7 & 14,3 & 0,0 & 21,0 \\
\hline 1RHZES/2RHZE/5(RHE)3 & $\begin{array}{l}\text { Lima Ciudad, Norte y } \\
\text { Sur, } 1996\end{array}$ & 84 & 58,3 & 17,8 & 10,7 & 8,3 & 4,7 & $S / I$ \\
\hline 3KCxEtZE/15CxEtZE & Perú 1997-1999 & 174 & 46,0 & 39,7 & 6,9 & 7,5 & 0,0 & $S / 1$ \\
\hline Individualizado & Perú 1997-2003 & $S / I$ & 64,4 & 2,2 & 21,0 & 12,0 & 1,8 & $S / 1$ \\
\hline Individualizado & Perú 2002-2003 & $S / I$ & 79,1 & 2,9 & 10,4 & 7,4 & 0 & $S / I$ \\
\hline \multirow[t]{14}{*}{$\begin{array}{l}\text { 4-6KCxEtCsZE/ 14- } \\
\text { 18CxEtCsZE }\end{array}$} & Perú 2000 ESNPCT & $S / I$ & 73,4 & 3,8 & 20,3 & 2,5 & 0,0 & $S / I$ \\
\hline & Perú 2001 ESNPCT & $S / I$ & 67,0 & 3,2 & 19,0 & 10,8 & 0,0 & $S / I$ \\
\hline & Perú 2002 ESNPC & 1423 & 65,3 & 4,5 & 17,2 & 13,1 & 0,0 & $S / I$ \\
\hline & Perú 2003 ESNPCT & 1679 & 66,2 & 3,4 & 15,5 & 14,9 & 0,0 & $\mathrm{~S} / \mathrm{I}$ \\
\hline & Perú 2004 ESNPCT & 1919 & 66,2 & 4,2 & 11,2 & 18,4 & 0,0 & $S / I$ \\
\hline & Perú 2005 ESNPCT & 2365 & 68,9 & 4,4 & 9,9 & 15,8 & 0,0 & $S / l$ \\
\hline & Perú 2006 ESNPCT & $S / 1$ & 66,2 & 6,7 & 8,4 & 18,1 & 0,0 & $S / 1$ \\
\hline & Perú 2007 ESNPCT & 1011 & 60,0 & 8,0 & 10,0 & 10,0 & 4,0 & $S / I$ \\
\hline & Perú 2008 ESNPCT & 1113 & 60,0 & 9,0 & 8,0 & 19,0 & 4,0 & $S / I$ \\
\hline & Perú 2009 ESNPCT & 1156 & 59,0 & 8,0 & 8,0 & 19,0 & 7,0 & $S / I$ \\
\hline & Perú 2010 ESNPCT & 1074 & 61,7 & 7,1 & 7,4 & 18,3 & 5,6 & $S / 1$ \\
\hline & Perú 2011 ESNPCT & $S / I$ & 57,0 & 4,6 & 7,6 & 27,6 & 3,2 & $S / I$ \\
\hline & Perú 2012 ESNPCT & $S / I$ & 60,5 & 2,9 & 6,0 & 29,8 & 0,8 & $S / 1$ \\
\hline & Perú 2013 ESNPCT & $S / I$ & 56,8 & 5,0 & 5,7 & 31,2 & 1,3 & $S / I$ \\
\hline
\end{tabular}

Se muestra las cohortes de tratamiento de tuberculosis nultirresistente (TBMDR) según año, esquemas y condición de egreso de las mismas, durante el periodo 1996 2013. Se observa que los esquemas de tratamiento han variado profusamente, desde un esquema UNO hasta retratamientos individualizados. Se observa también que la proporción de fracasos al tratamiento era elevado en los primeros años y en la última cohorte llego a 5,0\%. Se observa también como se incrementa progresivamente el abandono de tratamiento, llegando hasta un elevadísimo 31\% (uno de cada tres). ESNPCT: Estrategia Sanitaria Nacional para el Control de la Tuberculosis; S/I: sin información; Transf S/C:Transferido sin control.

TBMDR impactaron sobre la epidemia, llegando a detener la tendencia ascendente. Era de esperar que, en los años siguientes del 2011 para adelante, los casos anuales de TBMDR empezaran a decrecer, como ha ocurrido en todos los países exitosos. Sin embargo, nuevamente la TBMDR está incrementándose de manera sostenida, siendo los casos de TBXDR una proporción creciente de los TBMDR, próximo al 9\%. Probablemente algunas causas asociadas a este recrudecimiento puedan ser: el debilitamiento de las actividades de supervisión y capacitación de la ESNPCT, nuevamente la subestimación del problema de la TBMDR por parte de los médicos generales a cargo de la ESNPCT y de los organismos internacionales que han apoyado la gestión, sin advertir del deterioro del control de la TBMDR, la falta de acceso a tratamiento para TBXDR (utilizándose actualmente los esquemas para TBMDR), el debilitamiento del sistema de información y análisis, la presencia de cepas más virulentas, la falta de una política terapéutica eficaz hacia los contactos de los casos índices de TBMDR, periodos de interrupción de abastecimiento de medicamentos para TBMDR, etc. Tabla 1.

\section{Discusión}

Los resultados preliminares del último estudio nacional de prevalencia de resistencia a drogas anti TB del 2014-2015 (aún no publicado) muestran nuevamente una elevación significativa de la resistencia, incluyendo la TBMDR primaria o inicial. Otra vez repetimos los mismos errores de los años 1990: no necesitamos de cepas virulentas para crear una hiperendemia de TBMDR en Perú, bastó con el acúmulo de nuestros propios errores a gran escala durante largo tiempo. La información disponible aquí analizada sugiere que una serie de errores secuenciales en la 
apreciación de la magnitud, las causas, el impacto y la forma de abordaje para controlarla, así como la demora en la oportunidad para enfrentarla, han contribuido a una endemia de TBMDR en Perú, la más extensa de América, predisponiendo a la aparición de formas más peligrosas de TBMDR.

El programa de control de TB de Perú, siendo un programa exitoso en el manejo de TB simple o TB sensible, decidió alinearse con las recomendaciones de OMS acerca del abordaje de la TBDR, subestimando la magnitud de la epidemia y su transmisibilidad, la subestimación del papel del laboratorio en el control de la TBMDR y demorando en adquirir prontamente el tratamiento para el $100 \%$ de los casos. En aquellos años predominaba el concepto de que la TBMDR no era tan transmisible como la TB sensible y que bastaría con una buena estrategia DOTS con esquema acortado para controlarla. Lo ocurrido en Perú fue objeto de debate internacional pues no se explicaba cómo es que, siendo un programa modelo en el control de la TB sensible, ocurrió sin embargo, un incremento sostenido de la TBMDR, fenómeno que luego se sabría que ocurrió en otros países modelos.

Dos intervenciones acaecidas a partir de mediados de los años 2000 han demostrado ser exitosas: la universalización temprana de las pruebas de sensibilidad (sin esperar que fracase el paciente para recién ofertarla) y el acceso temprano a tratamiento para todos los pacientes con TBMDR. Sin embargo, el debilitamiento del programa en los últimos años ha revertido la disminución en curso de la endemia y otra ola sostenida de incremento está en progreso. Se requiere algunas intervenciones para adelantarse a la nueva diseminación en curso: 1) universalizar de una vez al $100 \%$ el acceso a las pruebas moleculares para detección temprana de TBMDR, lo cual implica descentralizar los laboratorios para pruebas moleculares (no es suficiente detectar TBMDR, se requiere detectar resistencia a isoniacida pues precede a la TBMDR); 2) fortalecer el liderazgo clínico de la UTTBMDR y de la ESNPCT para evitar las malas prácticas diagnósticas y terapéuticas en el manejo regional y local de la TBMDR; 3) mejorar urgentemente el sistema de información de TBMDR; 4) asegurar el acceso a tratamiento para TBMDR y TBXDR (no todos estos últimos están recibiendo el tratamiento apropiado); 5) fortalecer o retomar el programa de capacitación y supervisión de los niveles locales; 6) abordar decisiones sobre cómo tratar preventivamente a los contactos de casos índices con TBMDR; 7) acortar el tratamiento de TBMDR a 12-18 meses, con drogas potentes actualmente disponibles y en poblaciones con lesión radiológica mínima, con resistencia solo a drogas de primera línea, con conversión rápida y sin comorbilidad; 8) establecer una residencia sanitaria voluntaria para los pacientes con TBXDR, con incentivos económicos que contribuyan a la adherencia al tratamiento; 9) abordar el tema de soporte socioeconómico para las familias de afectados de TBMDR y TBXDR; 10) incrementar la oferta de habitaciones de aislamiento a presión negativa para los pacientes con TBMDR y TBXDR.

Agradecimientos: Dr Efraín Félix H, por la revisión y comentarios. Fuente de financiamiento: autofinanciado.

Conflictos de interés: Los autores declara no tener conflictos de interés.

\section{REFERENCIAS BIBLIOGRÁFICAS}

1. Pablos-Mendez A, Gowda DK, Frieden TR. Controlling multidrugresistant tuberculosis and access to expensive drugs: a rational framework. Bull World Health Organ. 2002;80(6):489-95.

2. Gillespie SH. Evolution of drug resistance in mycobacterium tuberculosis: clinical and molecular perspective. Antimicrob Agents Chemother. 2002;46(2):267-74.

3. Keshavjee S, Farmer PE. Tuberculosis, drug resistance, and the history of modern medicine. N Engl J Med. 2012;367(10):931-6.

4. Farmer $\mathrm{P}$, Bayona J, Becerra M, Furin J, Henry C, Hiatt $\mathrm{H}$, et al. The dilemma of MDR-TB in the global era. Int J Tuberc Lung Dis. 1998;2(11):869-76.

5. Mitnick C, Bayona J, Palacios E, Shin S, Furin J, Alcántara F, et al. Community-Based Therapy for Multidrug-Resistant Tuberculosis in Lima, Peru. N engl J Med. 2003;348(2):119-28.

6. Luelmo F. Informe de viaje al Perú. 10.05.1996. Ref: PER/ OCD/010/72/02/1261/96. Lima: OMS; 1996.

7. Dye C, Williams BG, Espinal MA, Raviglione MC. Erasing the world's slow stain: strategies to beat multidrug-resistant tuberculosis. Science. 2002;295(5562):2042-6.

8. World Health Organization. Multidrug-resistant tuberculosis - A human-made phenomenon. World TB Day 24 March 1997. Geneva: WHO; c1997.

9. Organización Mundial de la Salud. Directrices para el tratamiento de la tuberculosis farmacorresistente. WHO/TB96.210(Rev1) S. Ginebra: OMS; 1997.

10. World Health Organization. Report: Multidrug resistant tuberculosis (MDR TB). Basis for the development of an evidence-based casemanagement strategy for MDR TB within the WHO's DOTS strategy. Proceedings of 1998 meeting and protocol recommendations [Internet]. Geneva: WHO; c2017 [citado el 20 de marzo de 2017] Disponible en: http://www.who.int/docstore/gtb/publications/ mdrtb/PDF/who.tb.99.260.pdf

11. Yáñez $A$. Conceptos técnicos y operacionales actuales sobre el tratamiento de la tuberculosis. PNSP/93-09. Washington, DC: OPS; 1993.

12. World Health Organization. Policy guidance on TB drug susceptibility testing (DST) of second-line drugs (SLD). Expert Group Meeting. Geneva, Switzerland: WHO; 2007.

13. World Health Organization. Policy guidance on drug-susceptibility testing (DST) of second-line antituberculosis drugs. Geneva: WHO; 2008 [citado el 27 de diciembre de 2016]. Disponible en: http:// apps.who.int/iris/handle/10665/70500

14. Bastian I, Rigouts L, Van Deun A, Portaels F. Directly observed treatment, short-course strategy and multidrug-resistant tuberculosis: are any modifications required? Bull World Health Organ. 2000;78(2):238-51.

15. Suárez G. Boletín de Salud №19. Lima: Instituto de Salud; 2000.

16. Jave Castillo O. Tuberculosis multirresistente en Perú [diapositivas] [Internet]. Lima: Ministerio de Salud del Perú; 2005 [citado el 20 de marzo de 2017]. Disponible en: ftp://ftp2.minsa.gob.pe/descargas/ dgsp/ESN-tuberculosis/Exposiciones/TBMDRPeru2006.pdf

17. Vásquez-Campos L, Asencios-Solis L, Leo-Hurtado E, Quispe-Torres $\mathrm{N}$, Salazar-Lindo E, Bayona J, et al. Drug resistance trends among previously treated tuberculosis patients in a national registry in Peru, 1994-2001. Int J Tuberc Lung Dis. 2004;8(4):465-72.

18. Partners in Health. PIH's web-based medical records system earns headlines and awards [Internet]. Boston, MA: Partners In Health; 2006 [citado el 27 de diciembre de 2016]. Disponible en: http:// www.pih.org/blog/pihs-web-based-medical-records-system-earnsheadlines-and-awards

19. Blaya JA, Shin SS, Yagui MJ, Yale G, Suarez CZ, Asencios LL, et al. A web-based laboratory information system to improve quality of care of tuberculosis patients in Peru: functional requirements, implementation and usage statistics. BMC Med Inform Decis Mak. 2007;7:33

20. Blaya JA, Shin S, Contreras C, Yale G, Suarez C, Asencios L, et al. Full impact of laboratory information system requires direct use by clinical staff: cluster randomized controlled trial. J Am Med Inform Assoc. 2011;18(1):11-6. 
21. Blaya JA, Shin SS, Yale G, Suarez C, Asencios L, Contreras C, et al. Electronic laboratory system reduces errors in National Tuberculosis Program: a cluster randomized controlled trial. Int J Tuberc Lung Dis. 2010;14(8):1009-15.

22. Asencios L, Sanabria H, Diaz S., Quispe N. Resistencia inicial del M tuberculosis a los medicamentos antituberculosos (1988-1989). Rev Med Hered. 1993;4(2):62-66.

23. Asencios L., Sanabria H., Diaz S., Vasquez L., Quispe N. Resistencia primaria del Mycobacterium tuberculosis a los medicamentos anyituberculosis en el Perú, 1993-1994. Rev Peru Epidemiol. 1996;9(2):40-4

24. Kochi A, Vareldzis B, Styblo K. Multidrug-resistant tuberculosis and its control. Res Microbiol. 1993;144(2):104-10.

25. Hopewell PC, Ganter B, Baron RB, Sanchez-Hernandez M. Operational evaluation of treatment for tuberculosis. Results of 8- and 12-month regimens in Peru. Am Rev Respir Dis. $1985 ; 132(4): 737-41$.

26. World Health Organization. Anti-tuberculosis drug resistance in the world. Report N². Prevalence and trends. The WHO/IUATLD global project on anti-tuberculosis drug resistance surveillance. Geneva: WHO; 2000.

27. Aréstegui J, Molero G, Martínez G. Retratamiento de la tuberculosis pulmonar [Internet]. En: Seminario Taller Nacional: evaluación del programa de control de tuberculosis, año 1991-Perú. Lima: Ministerio de Salud del Perú; 1991. p. 47-9 [citado el 20 de marzo de 2017]. Disponible en: http://bvs.minsa.gob.pe/local/ minsa/362 PROG158.pdf

28. Oswaldo Jave C. Tuberculosis en el Perú. Retratamiento de tuberculosis con esquema KDRZE en dos zonas de Lima Norte. Informe 1995. Lima: Ministerio de Salud del Perú; 1995.

29. Jave Castillo O. Retreatment of MDRTB with 12 months 4KDRZE/8DRZE [abstract]. Int Jour Tuberc Lung Dis. 2001;5 Suppl.

30. Jave Castillo O. Del origen de la TBMDR en el Perú [diapositivas]. Lima: Sociedad Peruana de Medicina Interna; 2006.

31. Bonilla C, Sabogal I, Gutarra K. Tuberculosis en el Perú, 1994. Retratamiento con esquema de primera línea más suplemento de KNM y TB en pacientes con condición de fracaso. Lima: Ministerio de Salud del Perú; 1994.

32. Roberto Canales. Primera evaluación por estudio de cohorte de tratamiento diferenciado del esquema DOS 1RHZES/2RHZE/5(RHE)2. En: Ministerio de Salud del Perú. Tuberculosis en el Perú. Informe anual 1997. Lima: Ministerio de Salud del Perú; 1997. p. 55-9.

33. Ministerio de Salud del Perú. Situación de la Tuberculosis en el Perú [Internet]. Lima: MINSA; 2011 [citado el 20 de enero de 17]. Disponible en: https://www.minsa.gob.pe/ portada/Especiales/2011/respiravida/archivos/Ayuda_memoria_ Lanzamiento TB.pdf

34. Asian, C, Castilla T, Coyllo S, Jave O, Luis J, Suárez J. Tuberculosis en el Perú, año 1993. Estudio de cohorte retrospectiva de dos unidades territoriales de salud de Lima Norte, Perú. Lima: Ministerio de Salud del Perú; 1993.

35. Zavala D. Evaluación por estudio de cohorte de tratamiento del esquema uno. Primer semestre 1998. En: Ministerio de Salud del Perú: Tuberculosis en el Perú: informe 1998. Lima: Ministerio de Salud del Perú; 1998. p. 39-45.

36. A Harries. Chap 35. How does treatment of tuberculosis differ in persons infected with HIV? En: Toman. Case finding TB. Geneva: WHO; 1979. p. 169-72.

37. Directiva 006-1996-PCT Uso racional de los esquemas de tratamiento diferenciado en tuberculosis. Lima, Perú. MINSA 17/04/1996.

38. Espinal MA, Kim SJ, Suarez PG, Kam KM, Khomenko AG, Migliori $\mathrm{GB}$, et al. Standard Short -Course Chemotherapy for DrugResistant Tuberculosis. Treatment outcomes in 6 countries. JAMA. 2000;283(19):2537-45.

39. Blumberg HM, Burman WJ, Chaisson RE, Daley CL, Etkind SC, Friedman LN, et al. American Thoracic Society/Centers for Disease Control and Prevention/Infectious Diseases Society of America: treatment of tuberculosis. Am J Respir Crit Care Med. 2003;167(4):603-62.
40. Jave O. La tuberculosis multirresistente en el Perú. Cuaderno de Trabajo N²1. Lima: ForoSalud; 2003.

41. Vásquez L, Asencios L, Quispe N, Díaz S, Carrillo C, Portocarrero J, et al. Vigilancia de la resistencia a los medicamentos antituberculosos en el Perú. 1995-1996. Rev Med Exp INS. 1997;14(1):5-14.

42. Vigilancia de la resistencia a los medicamentos antituberculosos en el Perú. En: Ministerio de Salud del Perú: Tuberculosis en el Perú. Informe 1999. Lima: Ministerio de Salud del Perú; 1999. p. 483-92.

43. Asencios L, Quispe N, Mendoza-Ticona A, Leo E, Vásquez L, Jave 0 , et al. Vigilancia nacional de la resistencia a medicamentos antituberculosos, Perú 2005-2006. Rev Peru Med Exp Salud Publica. 2009;26(3):278-87.

44. American Thoracic Society/Centers for Disease Control and Prevention/Infectious Diseases Society of America: treatment of tuberculosis. Am J Respir Crit Care Med. 2003;167(4):603-62.

45. Directiva 017-1996-PCT. Uso Racional de la Prueba de sensibilidad. Lima, Perú. MINSA 17 de Septiembre de 1996.

46. Programa de control de tuberculosis. Vigilancia de la resistencia a los medicamentos antituberculosos en el Perú, 1999. En: Ministerio de Salud del Perú. Tuberculosis en el Perú. Informe 1999. Lima: Ministerio de Salud del Perú; 1999. p. 183-92.

47. Velásquez GE, Yagui M, Cegielski JP, Asencios L, Bayona J, Bonilla $\mathrm{C}$, Jave HO, et al. Targeted drug-resistance testing strategy for multidrug-resistant tuberculosis detection, Lima, Peru, 2005-2008. Emerg Infect Dis. 2011;17(3):432-40.

48. Ministerio de Salud del Perú. Norma Técnica. Estrategia Nacional de Salud de Tuberculosis. Lima: Ministerio de Salud del Perú; 2006.

49. Asencios L, Galarza M, Quispe N, Vásquez L, Leo E, Valencia E, et al. Prueba molecular Genotype ${ }^{\circledR}$ MTBDRplus, una alternativa para la detección rápida de tuberculosis multidrogorresistente. Rev Peru Med Exp Salud Pública. 2012;29(1):92-8.,

50. Jave O. MDRTB in Peru [Internet]. En: Curso Internacional de TBMDR. Lima: Green Light Committee; 2010 [citado el 25 de diciembre de 2016]. Disponible en: ftp://ftp2.minsa.gob.pe/ descargas/dgsp/ESN-tuberculosis/Informes/tbvision/TBMDR_ PERU.pdf

51. Jave O. La situación de la tuberculosis en Perú [Internet]. En: Presentación en "Foro A 5 años del pedido de emergencia TB". Lima: ISDEN; 2014 [citado el 25 de diciembre de 2016]. Disponible en: http://www.isden.org.pe/es/descarrr/ category/26-2014-11-19-17-49-15.html

52. Jave O. El tratamiento de la tuberculosis en el Perú. Revista Salud y Medicamentos. 2015;17(65):4-27.

53. El laboratorio en el control de la tuberculosis. En: Ministerio de Salud del Perú. Norma técnica de salud para el control de la tuberculosis. Lima: Ministerio de Salud del Perú; 2006.

54. Valentina Alarcón G. Situación de la Prevención y Control de la Tuberculosis en el Perú. Evaluación de los indicadores epidemiológicos, operacionales de Tuberculosis. Ministerio de Salud y ejecución presupuestal del PP 016 TB-VIH en Lima Metropolitana. I Sem 2016 San Borja, 20 de Julio del 2016.

55. Jave O. Situación de la TB y TBMDR en el Perú. En: Tuberculosis: Emergencia en Lima metropolitana. Lima, Perú: ISDEN; 2016.

56. Riekstina V, Sture I, Leimane V, Leimans J. Effective tuberculosis control in the setting of high level anti-tuberculosis drug resistance. EpiNorth. 2009;10(3):128-35.

57. Hollo V; National Tuberculosis Registry, Estonia. Tendencies of tuberculosis incidence in Estonia, 1998-2006. EpiNorth. 2007;11:48:39.

58. Magnitud del problema de TBMDR y cobertura de los tratamientos para TBMDR. En: Construyendo alianzas estratégicas para detener la tuberculosis: la experiencia peruana. Lima: Ministerio de Salud del Perú; 2006. p. 79-97.

59. Hinojosa EF. Título [abstract]. En: Conferencia Mundial de Tuberculosis y Enfermedades Respiratorias. Paris, Francia: International Union Against Tuberculosis and Lung Disease, Octubre 1992.

60. Ministerio de Salud del Perú. Evaluación de un grupo de pacientes con esquema de retratamiento estandarizado para TBMDR. Octubre 
1997 - diciembre 1998 [diapositiva]. Lima: Ministerio de Salud del Perú; 1998.

61. Componente de control de Tuberculosis. En: Ministerio de Salud del Perú. Plan nacional de control de la tuberculosis 2003-2005. Lima: Ministerio de Salud del Perú; 2002.

62. Capítulo VIII. Tratamiento de la tuberculosis. En: Ministerio de Salud del Perú. Actualización de la doctrina, normas y procedimientos para el control de la tuberculosis en el Perú, 2001. Lima: Ministerio de Salud del Perú; 2001.
63. JF Broekmans. Chap 8. Control strategies and programme management. En: Tuberculosis. Back to the future.Edited by John DH Porter \& Keith PWJ McAdam. Edit Wiley, 1993.

64. Hinojosa EF. Lineamientos para tratamiento de tuberculosis multirresistente. Enfermedades del Tórax. 1997;41(3).

65. Tratamiento de la tuberculosis. En: Ministerio de Salud del Perú. Norma técnica de salud para el control de la tuberculosis. Lima: Ministerio de Salud del Perú; 2006.

\section{Ahora puede enviar sus artículos para Acta Médica Peruana en nuestro Open Journal System:}

www.amp.cmp.org.pe

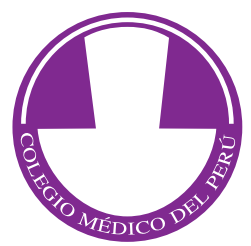

